\title{
Performance of OTDOA Positioning in Narrowband loT Systems
}

Kamiar Radnosrati, Gustaf Hendeby, Carsten Fritsche, Fredrik Gunnarsson and Fredrik Gustafsson

The self-archived postprint version of this journal article is available at Linköping University Institutional Repository (DiVA):

http:// urn.kb.se/ resolve?urn=urn:nbn:se:liu:diva-142819

N.B.: When citing this work, cite the original publication.

Radnosrati, K., Hendeby, G., Fritsche, C., Gunnarsson, F., Gustafsson, F., (2017), Performance of OTDOA Positioning in Narrowband IoT Systems, 28th Annual IEEE International Symposium on Personal, Indoor and Mobile Radio Communications (PIMRC).

https:// doi.org/ 10.1109/ PIMRC.2017.8292365

Original publication available at:

https:// doi.org/ 10.1109/PIMRC.2017.8292365

Copyright: IEEE

http://www.ieee.org/

(c) 2017 IEEE. Personal use of this material is permitted. However, permission to reprint/republish this material for advertising or promotional purposes or for creating new collective works for resale or redistribution to servers or lists, or to reuse any copyrighted component of this work in other works must be obtained from the IEEE. 


\title{
Performance of OTDOA Positioning in Narrowband IoT Systems
}

\author{
Kamiar Radnosrati, Gustaf Hendeby, Carsten Fritsche, Fredrik Gunnarsson ${ }^{\dagger}$, Fredrik Gustafsson \\ Department of Electrical Engineering, Linköping University, Linköping, Sweden \\ Email: \{firstname.lastname\}@liu.se \\ $\dagger$ Ericsson Research, Linköping, Sweden, Email: fredrik.gunnarsson@ericsson.com
}

\begin{abstract}
Narrowband Internet of Things (NB-IoT) is an emerging cellular technology designed to target low-cost devices, high coverage, long device battery life (more than ten years), and massive capacity. We investigate opportunities for device tracking in NB-IoT systems using Observed Time Difference of Arrival (OTDOA) measurements. Reference Signal Time Difference (RSTD) reports are simulated to be sent to the mobile location center periodically or on an on-demand basis. We investigate the possibility of optimizing the number of reports per minute budget on horizontal positioning accuracy using an on-demand reporting method based on the Signal to Noise Ratio (SNR) of the measured cells received by the User Equipment (UE). Wireless channels are modeled considering multipath fading propagation conditions. Extended Pedestrian A (EPA) and Extended Typical Urban (ETU) delay profiles corresponding to low and high delay spread environments, respectively, are simulated for this purpose. To increase the robustness of the filtering method, measurement noise outliers are detected using confidence bounds estimated from filter innovations.
\end{abstract}

\section{INTRODUCTION}

Although Global Navigation Satellite System (GNSS) solutions are capable of determining the position of an object with a few meters accuracy in outdoor environments, the robustness of GNSS-based methods is always restricted by the availability of GNSS signals. Indoor environments and dense urban areas are examples where these solutions fail.

As a response, the Third Generation Partnership Project Long Term Evolution (3GPP LTE) standard features positioning support since 3GPP Release 9. The subsequent releases, as explained in [1], further extended capabilities of positioning by introducing specific signaling infrastructures. For more information on positioning in LTE systems see [2]-[4].

The immense number of use cases inspired by IoT, however, motivated the 3GPP to introduce Release 14, NB-IoT. Wearable technologies, asset tracking, environmental monitoring are examples of 'things' addressed by IoT. Low power consumption and the possibility to communicate in the most challenging locations, in terms of coverage, are among shared requirements in all these scenarios. NB-IoT aims to offer deployment flexibility allowing an operator to allocate a small portion of its current available spectrum to NB-IoT. Coexistence performance with legacy Global System for Mobile Communications (GSM), General Packet Radio Service (GPRS) and LTE technologies is a primary design criterion for NB-IoT. As reported in [5], NB-IoT requires a minimum of $180 \mathrm{kHz}$ minimum system bandwidth for both downlink

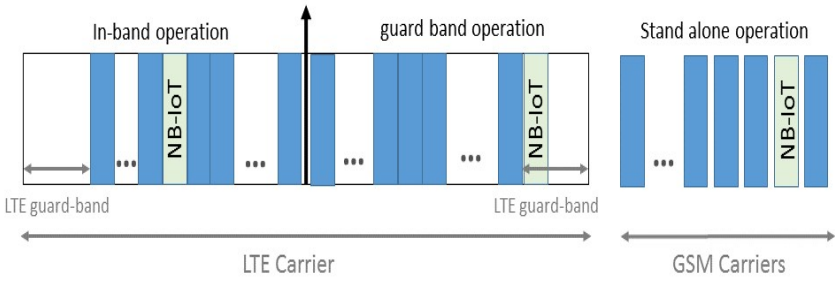

Fig. 1: Deployment configurations of NB-IoT.

and uplink. A GSM operator can replace one GSM carrier $(200 \mathrm{kHz})$ with NB-IoT. An LTE operator can deploy NBIoT inside an LTE carrier by allocating one of the Physical Resource Blocks (PRB) of $180 \mathrm{kHz}$ to NB-IoT.

The system used in this study simulates the deployment of NB-IoT within the LTE spectrum allocation, inside the LTE carrier. Other alternatives are to deploy the system as a stand-alone carrier or in the guard band of the LTE spectrum allocation. Fig. 1 illustrates all these alternatives of LTE deployments in the downlink.

To enhance Time Of Arrival (TOA) measurements precision, resulting in higher OTDOA positioning accuracy, LTE introduced the Positioning Reference Signal (PRS). NB-IoT systems are also equipped with the specific Narrowband PRS (NPRS) supporting downlink OTDOA based positioning. Specifically, for in-band deployment, PRS symbols are reused also in the NPRS, which typically is extended with a large number of repetitions to allow accumulation at the device for detectability.

OTDOA positioning in legacy LTE systems is widely studied in the literature. For example, [6] uses real measurements and investigates channel impacts on positioning accuracy. An error analysis of OTDOA is reported in [7]. Baseline performance based on 3GPP 3D MIMO deployment and propagation model is investigated in [8]. The work in [9] addresses the main requirement for accurate OTDOA positioning, synchronization of anchor nodes. Finally, surveys on the obtainable accuracy bounds are reported in [10], [8], and our previous work [11]. The recent NB-IoT systems, however, are not addressed extensively. This study investigates potential of device tracking in NB-IoT systems using OTDOA measurements with both snapshot and filtering algorithms. 
This paper is organized as follows. Section II-A presents NPRS transmission schemes compensating lower bandwidth of NB-IoT systems. Section II-B briefly describes the principle of RSTD estimation. Two position estimation algorithms based on snapshot optimization method and Extended Kalman Filter (EKF) based tracking filter are provided in Section II-B. Different RSTD reporting methods and other simulation scenarios are given in Section III. Section IV presents results for the considered scenarios followed by conclusions of the work in Section V.

\section{IOT POSITIONING IN LTE STANDARDIZATION}

Devices involved in IoT use cases require low-power consumption while demanding high positioning accuracies. They might be located indoors where GPS signals are not detectable or even might not support GPS or any other GNSS. Thus, the primary objective of NB-IoT is to provide a radio access technology that allows for low device complexity, with low power consumption while still providing an adequate throughput for the connected devices.

\section{A. NPRS Transmission schemes}

The reference signals used for TOA estimations are transmitted in so-called positioning occasions [12]. In LTE systems, in each positioning occasion $N_{\text {PRS }}$ consecutive subframes are sent every $T_{\mathrm{PRS}}$ subframe. In legacy LTE, $N_{\mathrm{PRS}}$ can be 1 , 2 , 4, or 6 , while $T_{\mathrm{PRS}}$ can be $160,320,640$ or $1280 \mathrm{~ms}$, see [13]. Fig. 2 shows an example of positioning occasion corresponding to a $20 \mathrm{MHz}$ LTE system with 100 Resource Blocks (RB). The bandwidth of each RB is $180 \mathrm{kHz}$ and the PRS, in this example, has $10 \mathrm{MHz}$ bandwidth. $\Delta_{\mathrm{PRS}}$ is the cell specific subframe offset which defines the starting subframe of the PRS transmission relative to the start of the system frame cycle.

For NB-IoT, the carrier bandwidth is $180 \mathrm{kHz}$, which fills up one LTE Physical Resource Block (PRB). To address the requirements of the IoT use cases, NB-IoT systems must ensure coverage and good performance in challenging indoor environments. Thus, denser NPRS transmissions compared to the legacy LTE PRS configuration are needed to support aggregation of data for adequate coverage.

Denser NPRS transmission occasion is achieved by extending $N_{\text {NPRS }}$ to also include $10,20,40,80$ or 160 subframes enabling even a continuous NPRS. Fig. 2 illustrates an example of NB-IoT NPRS transmission. Narrower bandwidth, as shown in the figure, can be compensated by longer $N_{\text {NPRS }}$. The bandwidth of NPRS is $180 \mathrm{kHz}$ equal to one PRB of the LTE. Supporting $N_{\text {NPRS }}=160$ subframes, it is possible to configure a transmission schedule to use all NB-IoT resources.

\section{B. RSTD Estimation}

The User Equipment (UE) measurement for OTDOA positioning is the Reference Signal Time Difference (RSTD) which is the relative time difference between the Evolved Node $\mathrm{B}$ (eNB) $j$ and the reference eNB $i$. RSTD is calculated as

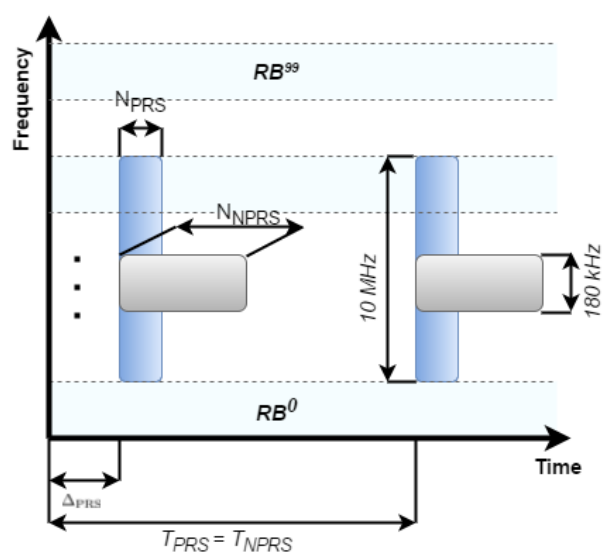

Fig. 2: Example of the PRS and NPRS transmission schemes.

the smallest time difference between two subframe boundaries received from two different eNBs.

In order to model the multipath behavior, we use the tapped delay line model for two LTE channel models. Multipath in Extended Pedestrian A (EPA) and Extended Typical Urban (ETU) is modeled as series of amplitude weighted delayed copies of the input signal. Let $L$ be the number of multipath channels with complex valued gains $\alpha_{\ell}$, the tapped delay line model is then given by

$$
h(t)=\sum_{\ell=0}^{L-1} \alpha_{\ell} \delta\left(t-\tau_{\ell}\right),
$$

where $\tau_{\ell}$ indicates the time delay of the $\ell^{\text {th }}$ tap and $\delta(t)$ is the Dirac delta function. Then, denoting the transmitted signal including OFDM modulated symbols and the cyclic prefix by $u$, the received version $r$ would be given by

$$
r(t)=u \star h(t)+\omega(t)
$$

where $\star$ stands for convolution and $\omega(t)$ is additive noise at the receiver. Let $\tilde{i}$ correspond to the discretized time $t$. The cross-correlation between the received sequence $r[\tilde{i}]$ and the reference sequence $u[\tilde{i}]$, denoted by $R(\tau)$ is computed per subframe. To consider $N_{\text {NPRS }}$ consecutive NPRS occasions, the average of the set of cross correlation estimates per positioning occasion is formed and denoted by $R_{\text {ave }}$. Finally, the first tap is estimated as

$$
\hat{\tau}=\underset{\tau}{\arg \min }\left\{\frac{R_{\text {ave }}(\tau)}{\max \left(R_{\text {ave }}\right)} \geq \zeta\right\}
$$

where $\zeta$ is the threshold value related to the multipath channel. The simulations of this paper use $\zeta=0.5$, which implies half of the strongest peak, see [8]. In order to estimate the position using the OTDOA method, each UE must be linked to RSTD measurements of multiple eNBs. Let $\hat{\tau}^{(\ell)}$ correspond to the TOA from the $\ell^{\text {th }}$ eNB to the UE. Then,

$$
\hat{\tau}^{(i, j)}=\hat{\tau}^{(i)}-\hat{\tau}^{(j)}
$$

where $\hat{\tau}^{(i, j)}$ is the RSTD between eNB $j$ and the reference eNB $i$. 


\section{Position Estimation}

The OTDOA method applies multilateration to estimate the position. Let the unknown UE's position in two-dimensional (2D) coordinates be denoted by $\mathbf{p}=\left(p_{x}, p_{y}\right)^{T}$. Further, let $\mathcal{N}$ be the set of eNBs in the cellular network; $\mathcal{N}=\{1, \ldots, N\}$. Then, for each $i \in \mathcal{N}$, the known location of the eNB is given by $\ell^{i}=\left(\ell_{x}^{i}, \ell_{y}^{i}\right)^{T}$. The Euclidean distance between the $i^{t h} \mathrm{eNB}$ and the UE is given by

$$
r^{i}=\left\|\mathbf{p}-\ell^{i}\right\|=\sqrt{\left(p_{x}-\ell_{x}^{i}\right)^{2}+\left(p_{y}-\ell_{y}^{i}\right)^{2}} .
$$

RSTD measurements can then be formed using the $K$ most powerful eNBs, measured in $\mathrm{dB}$, in the set $\mathcal{N}$. Hence, $K$ determines the number of eNBs used in the position estimation method. Let $\mathbf{h}_{\text {OTDOA }}(\mathbf{p})=r^{i}-r^{j},(i, j) \in K$, be the OTDOA measurement model. Further, $\mathbf{y}^{(i, j)}$ contains the RSTD reports in (4), translated to relative distances, of the $K$ most powerful eNBs,

$$
y^{(i, j)}=h_{\mathrm{OTDOA}}(\mathbf{p})+e^{(i, j)},
$$

where the additive error term $\mathbf{e}^{(i, j)}$ represents TOA measurement errors times the speed of light as well as NLOS propagation effects.

1) Static Case: In the static case, there is no assumption of temporal correlation between consecutive positions, so the position vector is a sequence of uncorrelated parameters estimated in a snapshot manner. The UE can be localized by finding the position that minimizes the cost function

$$
\hat{\mathbf{p}}=\underset{\mathbf{p} \in \mathbb{R}^{2}}{\arg \min }\left\|\mathbf{y}-\mathbf{h}_{\text {OTDOA }}(\mathbf{p})\right\|=\underset{\mathbf{p} \in \mathbb{R}^{2}}{\arg \min } f(\mathbf{p}) .
$$

Here, we use the modified Gauss-Newton method, as in [14], to find the minimizer of the cost function (7). Initialization is here chosen at the serving eNB. Then, each step is defined as,

$$
\begin{aligned}
\hat{\mathbf{p}}_{n}= & \hat{\mathbf{p}}_{n-1}+\alpha_{n}\left(\mathbf{H}^{T}\left(\hat{\mathbf{p}}_{n-1}\right) \mathbf{R}^{-1} \mathbf{H}\left(\hat{\mathbf{p}}_{n-1}\right)\right)^{-1} \\
& \mathbf{H}^{T}\left(\hat{\mathbf{p}}_{n-1}\right) \mathbf{R}^{-1}\left(\mathbf{y}-\mathbf{h}_{\mathrm{OTDOA}}\left(\hat{\mathbf{p}}_{n-1}\right)\right)^{T},
\end{aligned}
$$

where $\alpha_{n}$ is given by (9c) and $\mathbf{H}(p)=\nabla_{p} \mathbf{h}_{\mathrm{OTDOA}}(\mathbf{p})$ is the Jacobian matrix. Additive updates in each iteration, $\alpha_{n}$ are damped by using $\alpha_{n} \in(0,1]$ to avoid local convergence issues,

$$
\begin{aligned}
f_{n}\left(\alpha_{n}\right) & :=f\left(\hat{\mathbf{p}}_{n-1}+\alpha_{n} \Delta p\right) \\
M & :=\left\{\alpha_{n} \in\left\{1, \frac{1}{2}, \frac{1}{4}, \frac{1}{8}\right\}: f_{n}\left(\alpha_{n}\right)<f_{n}(0)\right\} \\
\alpha_{n} & =\left\{\begin{array}{cc}
\frac{1}{8} & \alpha_{n}=\varnothing \\
\max (M)) & \text { otherwise. }
\end{array}\right.
\end{aligned}
$$

where $\Delta p$ is the search direction equal to the one used in iterative updates in (8). RSTD measurements are performed with respect to a reference eNB, resulting in a correlated measurement noise covariance matrix R. Diagonal and offdiagonal elements of $\mathbf{R}$ are similar to the ones reported in [15], to capture spatial correlations.
2) Dynamic Case: The key idea with filtering is to include temporal correlation in a dynamic model, so that a prediction of the next position can be computed. Tracking filters can be applied to recursively estimate the state of dynamic systems from noisy measurements. The nonlinear function $\mathbf{f}$ transforms the state vector $\mathbf{x}$ at time step $k-1$ to the next time step $k$, while the measurement model $\mathbf{h}_{\text {OTDOA }}(\mathbf{x})$ relates the current state to the measurement $\mathbf{y}^{(i, j)}$,

$$
\begin{aligned}
& \mathbf{x}_{k}=\mathbf{f}\left(\mathbf{x}_{k-1}, \mathbf{w}_{k}\right) \\
& \mathbf{y}_{k}=\mathbf{h}_{\text {OTDOA }}\left(\mathbf{x}_{k}\right)+\mathbf{v}_{k},
\end{aligned}
$$

where the random variables $\mathbf{w}_{k}$ and $\mathbf{v}_{k}$ represent the noise of the state transition and the measurement, respectively. The noise is assumed to be white, mutually independent and normally distributed with covariances $\mathbf{Q}_{k}$ and $\mathbf{R}_{k}$, respectively.

This work uses $\mathbf{x}_{\mathbf{k}}=\left[p_{x, k}, p_{y, k}, v_{x, k}, v_{y, k}\right]$ where $v_{x, k}$ and $v_{y, k}$ denote the velocities in the $x$ and $y$ directions, respectively. In this work we use an Extended Kalman Filter (EKF). The process noise $\mathbf{w}_{k}$ is assumed to be Gaussian and additive. Denoting the $2 \times 2$ identity matrix $\mathbf{I}_{2}$ and the $2 \times 2$ zero matrix $\mathbf{0}_{2}$, the linearized discrete-time version of dynamic equation (10a) is then given by:

$$
\mathbf{x}_{k}=\left(\begin{array}{cc}
\mathbf{I}_{2} & T \mathbf{I}_{2} \\
\mathbf{0}_{2} & \mathbf{I}_{2}
\end{array}\right) \mathbf{x}_{k-1}+\left(\begin{array}{c}
\frac{T^{2}}{2} \mathbf{I}_{2} \\
T \mathbf{I}_{2}
\end{array}\right) \mathbf{w}_{k-1} .
$$

3) Outlier Detection: Measurement errors may contain outliers caused by NLOS and multipath effects. NPRS signals received from cells further away from the UE are more prone to channel imperfections. These signals, generally, have lower Signal to Noise Ratio (SNR) values. As shown in [8], the SNR of the links for RSTD reports, drastically affects TOA estimation error. For example, in order to have a TOA error less than 50 meters for $90 \%$ of the measurements, links must have SNR above $-10 \mathrm{~dB}$.

Fig 3 illustrates the TOA error in meters for the strongest cell for both channels EPA and ETU. The $90^{\text {th }}$ percentile of TOA errors for EPA and ETU channel models are around 30 and $300 \mathrm{~m}$, respectively. However, outliers of around $2 \mathrm{~km}$ can be seen in both channels.

Outliers from multipath effects are detected using hypothesis testing on measured residuals. Let $\hat{\mathbf{p}}_{k}$ to be the estimated position, the residual at time step $k$ is given by,

$$
\boldsymbol{e}_{k}=\mathbf{y}_{k}-\mathbf{h}_{\text {OTDOA }}\left(\hat{\mathbf{p}}_{k}\right) \text {, }
$$

The estimation covariance is given by $\mathbf{P}_{k}=\operatorname{Cov}\left(\hat{\mathbf{p}}_{k}\right)$. In the dynamic case, for example, $\mathbf{P}_{k}=\mathbf{P}_{k \mid k-1}$ denotes the one-step ahead prediction of the state covariance. In absence of outliers, $e_{k}$ is normally distributed,

$$
\boldsymbol{e}_{k} \sim \mathcal{N}\left(\mathbf{0}, \mathbf{H}_{k} \mathbf{P}_{k} \mathbf{H}_{K}^{T}+\mathbf{R}_{k}\right),
$$

Residuals outside the $95^{\text {th }}$ percentile of the estimated uncertainty ellipse are assumed outliers. 


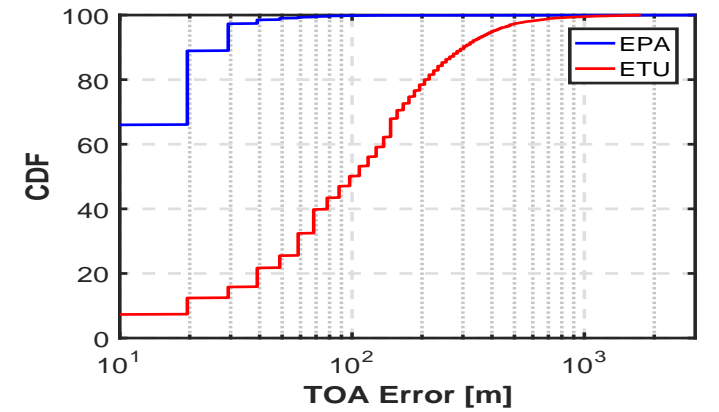

Fig. 3: CDF for TOA error in meters for strongest link, corresponding to the serving cell.

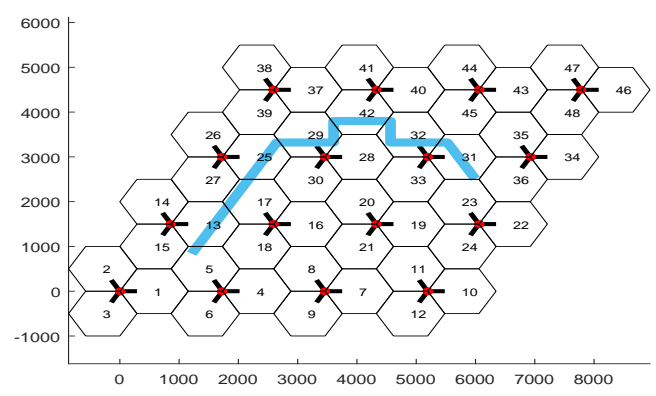

Fig. 4: Deployment configurations of NB-IoT. The blue line represents the UE's trajectory

\section{Simulation STUDY}

\section{A. Network Deployment}

The simulated cellular network consists of 16 macro sites each having 3 cells, located in a hexagonal grid with an intersite distance of $577 \mathrm{~m}$. The mobile UE starts at a random point in the cellular network and passes through the network with different speeds. Fig. 4 illustrates the cellular network deployment in which the red dots are macro sites, each with three cells, and the blue line represents the UE's trajectory. The UE trajectory represents a typical asset tracking use case, which is one important mobile IoT use case. The radio distance wrapping technique, as described in [8], is used in this work. This technique simulates a network in which all sites are assumed to be surrounded by other sites. That is, all the UEs that might be on the border of the deployed network in Fig. 4 are assured to be covered by other sites.

\section{B. NPRS Transmission and RSTD Reports}

As described in Sec. II-A, NB-IoT supports a denser NPRS transmission scheme allowing aggregation of data. This enhances TOA detection accuracy compared to other narrowband systems. The NB-IoT system with in-band configuration, as shown in Fig. 1, within the $20 \mathrm{MHz}$ LTE standard is simulated in this work. The NPRS bandwidth is $180 \mathrm{kHz}$ where $N_{\mathrm{NPRS}}=40$ subframes are transmitted every $T_{\mathrm{NPRS}}=160$ ms. The simulated NB-IoT system parameters are given in Table I. Based on the geometry of the user, hearability of cells
TABLE I: The deployed NB-IoT parameters.

\begin{tabular}{ll}
\hline & Deployed NB-IoT \\
\hline System carrier bandwidth & $20 \mathrm{MHz}$ \\
NB-IoT configuration & In-band \\
NB-IoT carrier bandwidth & $180 \mathrm{KHz}$ \\
Number of consecutive NPRS & 40 \\
NPRS period & $160 \mathrm{~ms}$ \\
\hline
\end{tabular}

and channel conditions, we form the SNR vector containing signal strengths received from each cell. The cells below a given threshold are assumed out of coverage and the $K$ remaining ones with highest SNR form $N_{\text {rep }}=K-1$ TDOA measurements. In this setup, the reference cell is the one with highest SNR value.

Let $T_{\text {rep }}$ be the interval at which the UE reports RSTD measurements to the positioning center. In periodic reporting, the UE forms $N_{\text {rep }}$ RSTD estimates and reports it to the positioning center periodically every $T_{\text {rep }}$ seconds. Alternatively, in the on-demand reporting method, the UE decides the number of RSTDs depending on the SNR of the measured cells. In cases where the UE receives NPRS signals from strong cells, it forms more RSTDs to increase the accuracy. Weaker signals are assumed to correspond to the eNBs further away from the UE that are more prone to severe measurement errors. In cases where the received signals are not strong enough, the UE reports less cells to lower the resource consumption.

In addition to the quantity of RSTD reports, the geometry of measuring cells also matters. In cases where the most powerful NPRS signals correspond to cells which are co-located on one site, they add no more information to the position estimation process. One remedy to co-located cell situations is to use the 28-bit cell identity information to provide reports of cells corresponding to different sites. In this work, the simulated UE employs this information to form and report RSTDs from unique sites, if possible. This is not always achievable, however, as there are cases in which hearable cells are very few and selecting signals from unique sites is not possible.

\section{Positioning Scenarios}

A well known technique to model a wireless channel in LTE is to use a (Finite Impulse Response) FIR filter. The wireless channel thus corresponds to a convolution of the transmitted signal. The channel profile quantifies the delays and relative powers of the multipath components as described in Sec. II-B.

Performance of the two positioning methods introduced in Sec. II is evaluated in terms of the Root Mean Square Error (RMSE).

1) Static Case: In the static case, we evaluate the obtainable position estimation accuracy using NPRS signals as a function of the number of measured cells.

2) Dynamic Case: In this case we consider three different UE speeds 3,10 , and $30 \mathrm{~km} / \mathrm{h}$. The reporting budget per minute $\mathcal{B}$ is defined as $\mathcal{B}=\frac{60}{T_{\text {rep }} \times N_{\text {rep }}}$. Reports are either sent periodically or on an on-demand basis forming five different cases:

- Case 1: Two reports every two seconds, $\mathcal{B}=60$. 
- Case 2: Three reports every three seconds, $\mathcal{B}=60$.

- Case 3: Five reports every four seconds, $\mathcal{B}=75$.

- Case 4: The UE increases the accuracy by forming more RSTD reports if SNR values of available cells are above a certain threshold. However, it cannot exceed a maximum budget of $\mathcal{B}_{\max }=85$.

- Case 5: Varying number of reports every four seconds. The UE has a maximum budget $\mathcal{B}_{\max }=45$ and depending on the SNR values, reports less or more cells to optimize resource consumption.

\section{PERformance Evaluation}

The positioning methods described in Sec. II are numerically evaluated and the results are presented by the cumulative distribution function of the RMSE of the horizontal accuracy.

Fig. 5 illustrates the $\mathrm{CDF}$ of the horizontal positioning accuracy of the OTDOA estimates for the static case for the EPA and the ETU channel models, separately. Each curve is the result of 50 Monte Carlo (MC) simulation runs.Although the maximum positioning error is the same for both channels, the $90^{\text {th }}$ percentile of the error with 4 cells is around 76 meters with the EPA channel and 495 meters with the ETU. Large positioning errors in Fig. 5 can be explained by multiple factors. There might be situations where less than three cells are heard. Although this can be handled using a tracking filter, the optimization method in the static case fails to converge as no unique optimum exists. NPRS signals received from cells belonging to same site is another major influencing factor. Although it is mentioned that the UE uses measurements from unique sites, this is not always possible as there are situations when 3 unique sites are not within range. In cases where the serving cell, initial point, is far away from the true position, the final estimate can be a local minimum of the cost function. In the snapshot positioning method, where no temporal correlation is considered, good initialization is more important.

Fig. 6 illustrates how poor initialization can be handled using tracking. The UE speed is $30 \mathrm{~km} / \mathrm{h}$ and RSTDs are sent as in case 1 . The shaded areas are the range of positioning errors obtained in $50 \mathrm{MC}$ runs and solid lines are the average positioning error. Fig. 7 presents the performance of the periodic reporting methods, cases 2 and 3, introduced in Sec. III for different UE speeds. It is seen that in EPA channel models, more frequent reporting with less cells gives better results for all different speeds. Cases 4 and 5 are compared to the periodic reporting for all cases and the results are presented in Table II. For example, in the ETU channel, for a UE moving with 30 $\mathrm{km} / \mathrm{h}$, case 4 improves the $90^{\text {th }}$ percentile of positioning error from $238 \mathrm{~m}$ in case 3 to 183 meters as seen in Fig. 8b. Case 5 on the other hand, reduces reports per minute budget to almost a half while giving almost the same error characteristics.

\section{CONCLUSION}

The performance of device tracking in NB-IoT systems has been investigated with respect to the horizontal positioning accuracy. The new transmission scheme of NPRS was used to

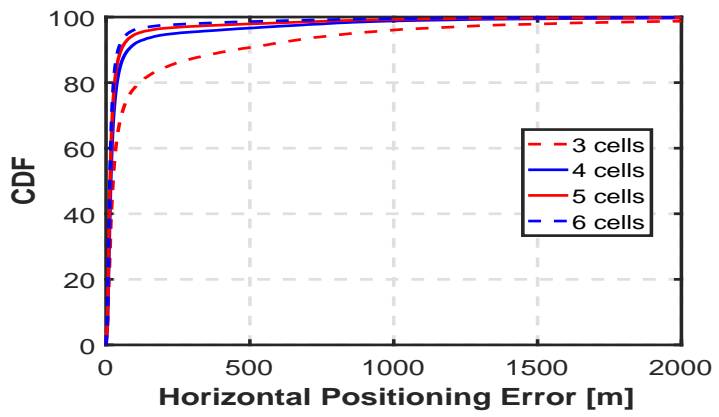

(a) Channel: EPA

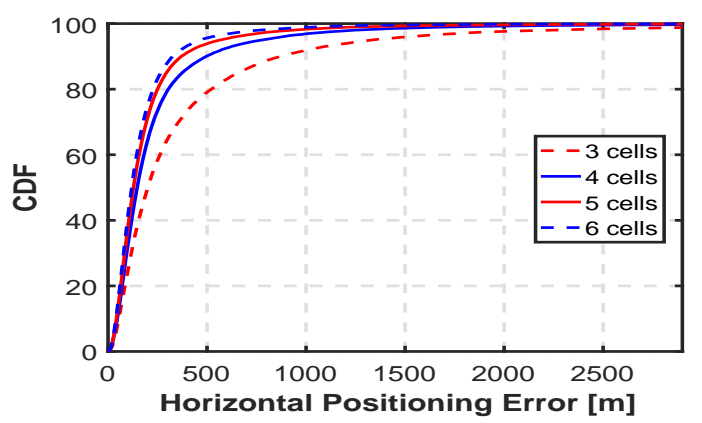

(b) Channel: ETU

Fig. 5: The OTDOA performance for horizontal position accuracy of Static case for both channel models.

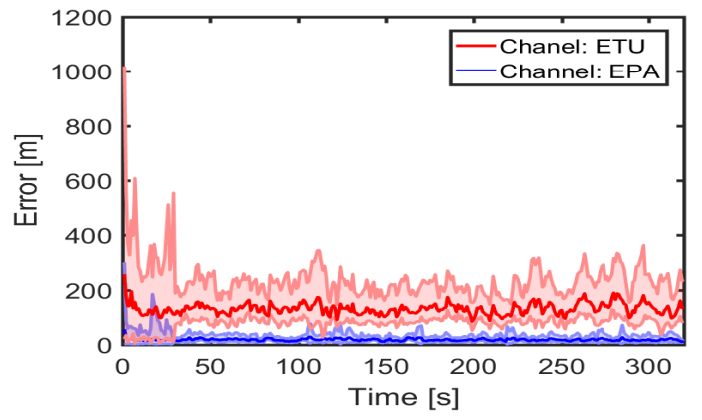

Fig. 6: RMSE over time for NB-IOT positioning using EKF for case 1 .

compensate narrower bandwidth of the system and improve TOA estimates. Given a certain NPRS transmission scheme, the RSTD was formed and used for downlink OTDOA positioning. The results were obtained in the EPA and ETU wireless channel models corresponding to low and high delay spread environments, respectively. We evaluated positioning in both static and dynamic cases. The measurement outliers were detected in the dynamic case, using the EKF innovations, resulting in a more robust algorithm.

\section{ACKNOWLEDGEMENT}

The authors would like to thank Dr. S. M. Razavi and H. Rydén at Ericsson Research, Linköping, Sweden for their valuable advices. This work is funded by the European Union 
TABLE II: Positioning error statistics and reporting budget $\mathcal{B}$ obtained by EKF for UE speeds $3 / 10 / 30 \mathrm{~km} / \mathrm{h}$.

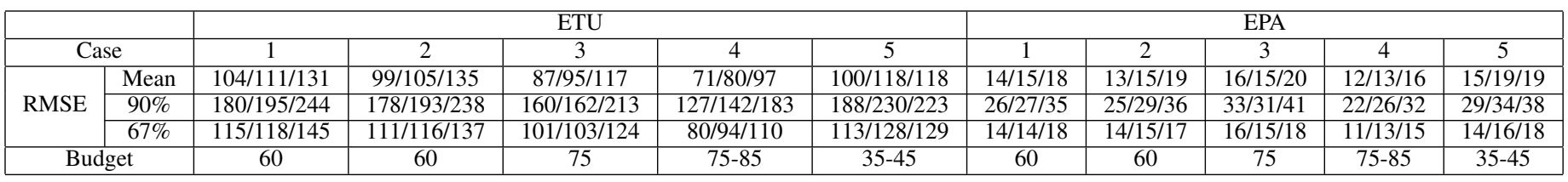

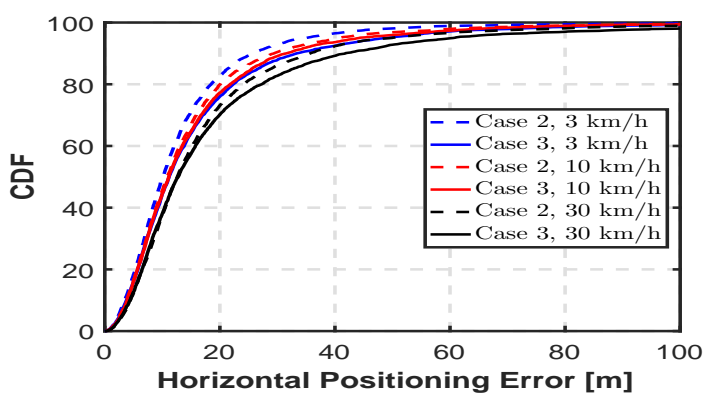

(a) Channel: EPA

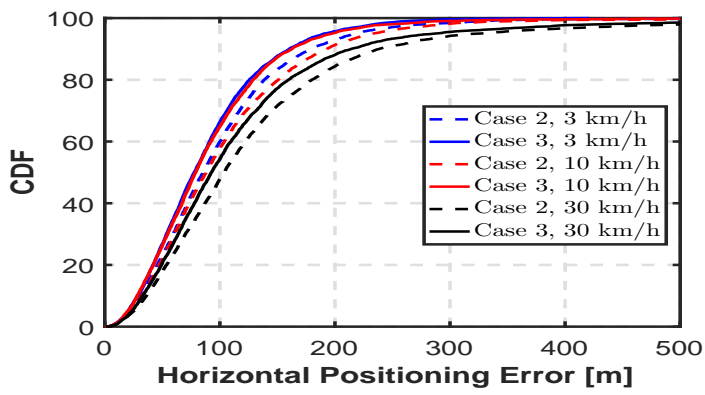

(b) Channel: ETU

Fig. 7: The OTDOA performance for horizontal position accuracy of EKF for both channel models.

FP7 Marie Curie training program on Tracking in Complex Sensor Systems (TRAX).

\section{REFERENCES}

[1] 3GPP TR 36.355, "Evolved Universal Terrestrial Radio Access (EUTRA); LTE Positioning Protocol (LPP)," Release 12.

[2] A. Kangas, I. Siomina, and T. wigren, Positioning in LTE. John Wiley \& Sons, Inc., 2011, pp. 1081-1127.

[3] M. Driusso, C. Marshall, M. Sabathy, F. Knutti, H. Mathis, and F. Babich, "Vehicular position tracking using LTE signals," IEEE Transactions on Vehicular Technology, vol. 66, no. 4, pp. 3376-3391, Apr. 2017.

[4] C. Y. Chen and W. R. Wu, "Three-dimensional positioning for LTE systems," IEEE Transactions on Vehicular Technology, vol. 66, no. 4, pp. 3220-3234, Apr. 2017.

[5] Y. P. E. Wang, X. Lin, A. Adhikary, A. Grovlen, Y. Sui, Y. Blankenship, J. Bergman, and H. S. Razaghi, "A primer on 3GPP narrowband internet of things," IEEE Communications Magazine, vol. 55, no. 3, pp. 117-123, Mar. 2017.

[6] J. Medbo, I. Siomina, A. Kangas, and J. Furuskog, "Propagation channel impact on LTE positioning accuracy: A study based on real measurements of observed time difference of arrival," in Proc. of the 20th International Symposium on Personal, Indoor and Mobile Radio Communications, Tokyo, Japan, Sep. 2009.

[7] S. H. Kong and B. Kim, "Error analysis of the OTDOA from the resolved first arrival path in LTE," IEEE Transactions on Wireless Communications, vol. 15, no. 10, pp. 6598-6610, Oct. 2016.

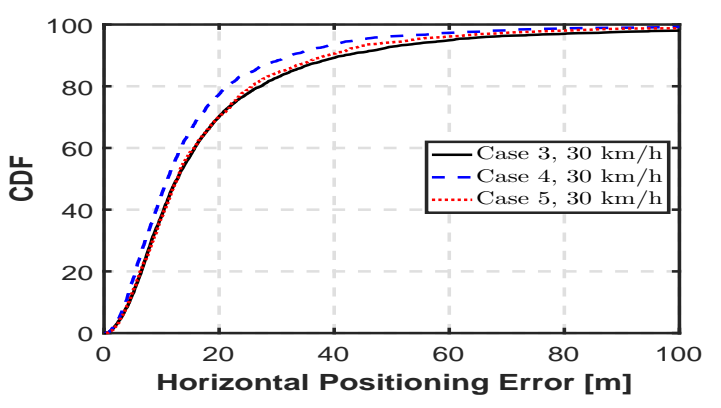

(a) Channel: EPA

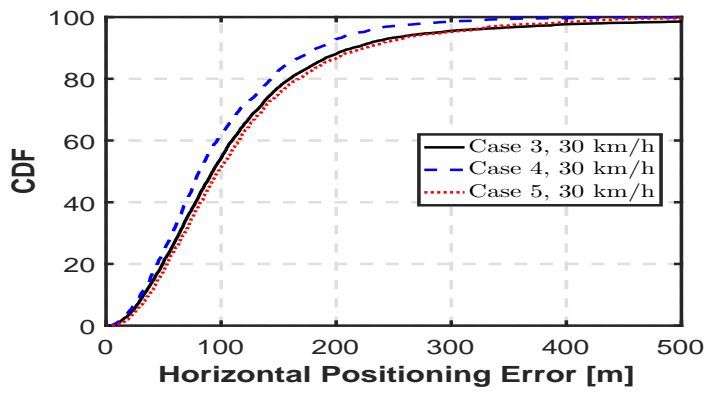

(b) Channel: ETU

Fig. 8: Comparison of on-demand with periodic reporting schemes.

[8] H. Rydén, S. M. Razavi, F. Gunnarsson, S. M. Kim, M. Wang, Y. Blankenship, A. Grövlen, and Å. Busin, "Baseline performance of LTE positioning in 3GPP 3D MIMO indoor user scenarios," in Proc. of the International Conference on Location and GNSS (ICL-GNSS), Gothenburg, Sweden, Jun. 2015.

[9] Z. Li, D. C. Dimitrova, D. H. Raluy, and T. Braun, "TDOA for narrowband signal with low sampling rate and imperfect synchronization," in Proc. of the 7th IFIP Wireless and Mobile Networking Conference (WMNC), Vilamoura, Portugal, May 2014.

[10] F. Gustafsson and F. Gunnarsson, "Mobile positioning using wireless networks: possibilities and fundamental limitations based on available wireless network measurements," IEEE Signal Processing Magazine, vol. 22, no. 4, pp. 41-53, Jul. 2005.

[11] K. Radnosrati, F. Gunnarsson, and F. Gustafsson, "New trends in radio network positioning," in Proc. of the 18th International Conference on Information Fusion (Fusion), Washington DC, USA, Jul., 2015.

[12] S. Fischer, "Observed time difference of arrival (OTDOA) positioning in 3GPP LTE," Qualcomm Technologies, Inc., 2014.

[13] 3GPP TS 36.211, "Physical channels and modulation," Release 12.

[14] C. Lass, D. A. Medina, I. D. H. Pinzón, and R.Ziebold, "Methods of robust snapshot positioning in Multi-Antenna systems for inland water applications," in Proc. of the European Navigation Conference, Helsinki, Finnland, May 2016

[15] R. Kaune, J. Hörst, and W. Koch, "Accuracy analysis for TDOA localization in sensor networks," in Proc. of the 14th International Conference on Information Fusion, Chicago, IL, USA, Jul. 2011. 